\title{
Fait/événement - Document/monument
}

La temporalité à l'œuvre

\section{Giuseppe Di Liberti}

\section{OpenEdition}

Journals

Édition électronique

URL : https://journals.openedition.org/imagesrevues/3319

DOI : 10.4000/imagesrevues.3319

ISSN : 1778-3801

Éditeur :

Centre d'Histoire et Théorie des Arts, Groupe d'Anthropologie Historique de l'Occident Médiéval, Laboratoire d'Anthropologie Sociale, UMR 8210 Anthropologie et Histoire des Mondes Antiques

\section{Référence électronique}

Giuseppe Di Liberti, «Fait/événement - Document/monument », Images Re-vues [En ligne], 11 | 2013, mis en ligne le 07 janvier 2014, consulté le 01 février 2022. URL : http://journals.openedition.org/ imagesrevues/3319; DOI : https://doi.org/10.4000/imagesrevues.3319

Ce document a été généré automatiquement le 1 février 2022.

\section{(c) (i) (8)}

Images Re-vues est mise à disposition selon les termes de la Licence Creative Commons Attribution Pas d'Utilisation Commerciale 4.0 International. 


\section{Fait/événement - Document/ monument}

La temporalité à l'œuvre

Giuseppe Di Liberti

Avant-propos 
1 Implicitement ou explicitement, toute description d'œuvre d'art rencontre inéluctablement une question-limite: jusqu'à quel point les catégories employées par le discours sur l'œuvre sont-elles aussi des catégories actives dans l'œuvre et constitutives de celle-ci ? D'une part, on pourrait dire qu'il s'agit d'une question épistémologique générale que n'importe quel discours scientifique et disciplinaire se pose face à ses objets. D'autre part, les œuvres d'art, comme tous les objets culturels et sociaux, posent un problème supplémentaire : leur nature dépend en partie du discours sur l'art. En d'autres termes, le questionnement ontologique est bien difficilement dissociable de l'épistémologie des disciplines qui construisent des discours

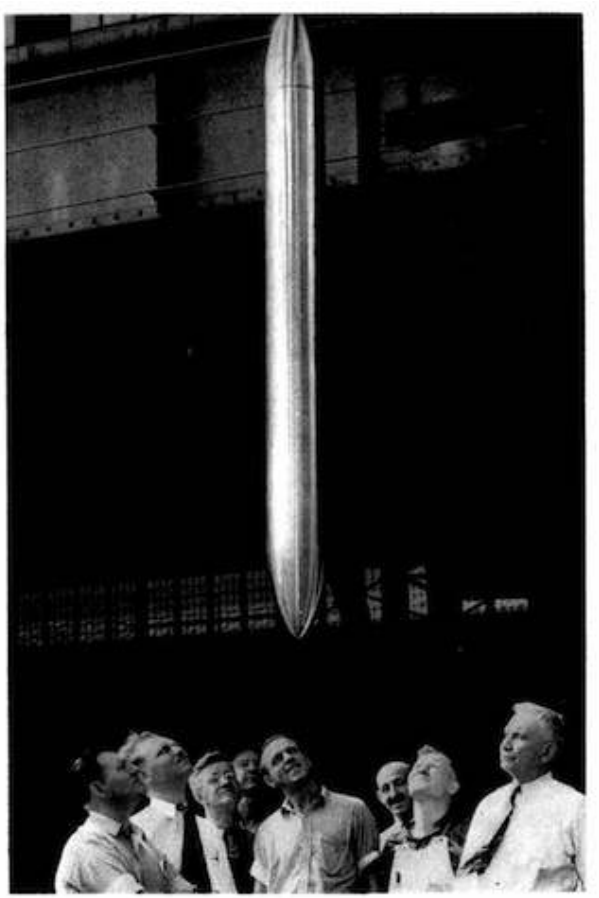
sur l'art, car même l'évolution des formes relève aussi des changements du discours (et vice versa, bien évidemment) ${ }^{1}$.

2 Même dans sa généralité, cette remarque introductive est nécessaire dès lors que nous cherchons à saisir la temporalité d'une œuvre d'art, car ce temps est aussi le temps de l'expérience esthétique que nous faisons de l'œuvre et à partir de laquelle nous débutons tout discours.

3 L'hypothèse que je voudrais ici proposer est la suivante : sur le plan ontologique, la coexistence de deux dimensions temporelles distinctes - celles du fait et de l'évènement - pourrait partiellement caractériser l'œuvre d'art en tant que telle. Sur le plan descriptif, nous pouvons considérer le couple fait/événement comme une polarité active dans l'objet artistique, qui nous permet de reconnaître une articulation du temps propre à l'œuvre d'art singulière.

4 La polarité fait/événement ne se présente jamais pure et chaque œuvre - comme chaque expérience de notre vie - instaure une relation nouvelle entre ce qui se passe ou ce qui est arrivé (les événements) et des versions des choses, des relations entre les éléments, des points de vue, des manières de décrire (les faits). En outre, elle est une polarité parmi d'autres polarités, qui ont entre elles des relations systémiques. À travers le couple fait/évènement, nous pouvons partiellement décrire la dimension temporelle d'une œuvre d'art et préciser davantage d'autres notions qui articulent le temps (et notamment le rapport entre le temps et la mémoire) de l'œuvre, telles que les notions de document et de monument.

Cette hypothèse nécessite quelques précisions méthodologiques préliminaires. 


\section{Quatre précisions}

6 La première: lorsque j'emploie le terme "polarité" pour discuter du couple fait/ événement, je fais référence au débat des années 1920 autour d'une systémique des concepts fondamentaux de la science de l'art et en particulier, aux réflexions d'Erwin Panofsky ${ }^{2}$ et d'Edgar Wind ${ }^{3}$ et à leur tentative de réorganiser l'approche des concepts fondamentaux de l'histoire de l'art de Riegl ${ }^{4}$ et de Wölfflin ${ }^{5}$. Même si je ne reviendrai que marginalement sur ce débat, j'envisage une possible incorporation du couple fait/ événement dans une systémique plus large des problèmes artistiques ${ }^{6}$.

7 La deuxième précision concerne une critique implicite de la tendance à une relative réduction de l'objet artistique à sa dimension représentative. Au contraire, considérer l'objet artistique sous l'angle de la polarité fait/événement signifie souligner sa dimension performative. Ainsi une image pourra ici être pensée comme un objet qui agit dans l'espace et dans le temps ${ }^{7}$.

8 Cette dimension nécessite une troisième précision : la difficulté de séparer l'eccéité de l'objet artistique de sa quiddité. Il y a une douzaine d'années, Joseph Margolis a adressé des critiques robustes à certains points clés des réflexions de Goodman et de Danto sur l'œuvre d'art ${ }^{8}$. Margolis mettait en évidence deux risques majeurs. Le premier concernait une symptomatologie (les fameux symptômes esthétiques de Goodman, par exemple mais aussi les indiscernabilité perceptuelles de Danto ) ignorant la maladie (un concept, même provisoire ou général, d'art) ${ }^{9}$. De manière implicite, Margolis critiquait les stratégies élusives de Goodman et Danto: parler des symptômes esthétiques sans éclaircir la nature de l'art, introduit une confusion entre l'analyse des conditions de manifestation des objets artistiques et la compréhension de la nature de ces objets et de leurs qualités. Le second risque portait sur la réduction de la perception à la sensation (Danto a construit son argument sur l'indiscernabilité perceptive entre une œuvre d'art et un objet quelconque, alors qu'il s'agit, selon Margolis, d'une simple indiscernabilité sensorielle). Avant tout, la perception "esthétique" n'est pas, dans la réflexion de Margolis, une perception "spéciale", mais plutôt un emploi spécial de la perception ${ }^{10}$. Ensuite, la perception est déjà "théoriquement chargée " et "culturellement et intentionnellement informée $»^{11}$, ce qui implique une dimension historique de la perception. Il s'agit là d'une conséquence de l'idée - radicalisée chez Margolis - du sujet comme construction historique. Cette radicalisation a le mérite d'établir une profonde affinité ontologique entre homme et œuvre d'art : autant que les hommes, les œuvres d'art sont des entités matériellement incorporées et culturellement émergentes, des objets physiques particuliers qui incorporent des types dont l'existence dépend justement de leur exemplification par un token ${ }^{12}$.

Or, la compréhension d'un phénomène aussi complexe qu'un objet artistique nécessite une recherche tant sur l'eccéité que sur la quiddité, que nous ne pouvons en aucun cas réduire l'une à l'autre. L'eccéité peut - ou plutôt "doit", à partir du moment où l'on choisit comme point de départ l'expérience que nous faisons des objets - précéder la quiddité, dans le sens qu'avant de comprendre la nature d'un objet il faudra l'identifier et comprendre les conditions de cette identification. Mais les conditions de présentification et d'identification de l'objet ne nous disent pas grand chose sur les raisons de son existence et sur ce que nous pouvons faire avec l'objet une fois que nous l'avons reconnu. Pour reprendre les termes de Goodman ${ }^{13}$, certes il est important de voir ce que l'art fait, mais il est également important de considérer ce que nous faisons 
avec l'œuvre. Et cette interaction doit être basée sur un terrain commun entre le discours de l'objet artistique et le discours sur l'objet artistique, terrain qui peut être déterminé par un système de polarités et qui est, au bout du compte, celui de l'expérience. Est-ce qu'une ontologie de l'œuvre d'art peut s'arrêter aux conditions visibles - de sa construction sans interroger la nature de cette construction? Les symptômes et les dispositifs de la représentation nous permettent de reconnaître un objet artistique, bien sûr. Mais qu'est-ce que nous re-connaissons ? Et surtout qu'est-ce qui se passe une fois l'objet reconnu ? La polarité fait/événement nous incite à penser l'œuvre comme mise en forme d'une expérience (perceptive et historique) et surtout comme forme d'expérience. Si nous pouvons admettre que la représentation et ses dispositifs nous permettent la reconnaissance, c'est avec la performance de l'œuvre que nous parvenons à une connaissance sensible.

Je passe maintenant à la quatrième et dernière précision. J'emploie dans cette recherche des outils théoriques propres à l'analyse du langage ordinaire et aux débats sur la notion de vérité qui en découlent. Je chercherai à manipuler ces outils avec discrétion. Il ne s'agit pour moi ni d'une réduction du phénomène artistique au signe, ni de la métaphore - souvent abusive - du langage de l'art ou des langages des arts. Il s'agit, encore une fois, de mettre au centre l'expérience comme expérience de la forme et l'action comme action de mise en forme. Les instruments d'analyse du langage ordinaire, surtout dans leurs développements après Austin et notamment chez Strawson, Cavell et Searle, nous offrent la possibilité de travailler une ontologie des objets culturels complexes où perception et nature de l'objet sont relativement coïncidentes. Je me permets aussi de renvoyer encore aux derniers travaux de Joseph Margolis, en particulier The Cultural Space of the Arts and the Infelicities of Reductionism ${ }^{14}$ de 2010, la nouvelle édition de Culture and Cultural Entities ${ }^{15}$ de 2009 et The Arts and the Definition of the Human: Toward a Philosophical Anthropology ${ }^{16}$ de 2008, pour indiquer la piste dans laquelle je voudrais inscrire ce travail. En résumé, il s'agit d'affirmer les points suivants: un pragmatisme qui refuse les réductionnismes, la tentative de dépasser l'opposition analytique/continentale, une profonde réévaluation de l'historicisme et de la "nature" historique de la pensée, une idée de l'humain comme construction culturelle et donc une affinité ontologique entre objet artistique et sujet humain.

\section{Les faits et les événements}

Il faudrait avant tout préciser, même de manière assez générale, la distinction entre faits et événements.

Au sens large, les événements sont des choses qui arrivent ${ }^{17}$. Comme les objets, ils sont des individus, dans le sens qu'ils occupent un segment d'espace et de temps. Mais, lorsque les objets sont dans le temps, dans la durée avec une relative stabilité, et qu'ils peuvent se déplacer dans l'espace, les événements ont lieu, se produisent dans le temps, dans une durée limitée et ils ne peuvent pas se déplacer. Quine ne fait pas une grande différence ontologique entre objets et événements car il les considère comme faits de la même matière habitant de l'espace-temps ${ }^{18}$, et souligne seulement une différence de vitesse entre objets (plutôt lents) et événements (plutôt rapides). Il y a un grand nombre de classifications des types d'événements, que je me permets de regrouper dans deux grandes catégories, en me référant à Ingarden ${ }^{19}$ : les processus (c'est-à-dire des 
événements homogènes) et les achèvements (c'est-à-dire des événements ponctuels, instantanés). Ensuite, ces événements peuvent être physiques ou mentaux, intentionnels ou non intentionnels, mais ces questions risquent de nous emmener trop loin.

Les faits sont normalement bien distincts des événements, car leur nature est abstraite et atemporelle ${ }^{20}$. Pour préciser : la validité - les conditions de vérité - d'un fait est abstraite et atemporelle, mais les conditions de sa formulation et de son énonciation sont bien historiques. Un fait est affirmé par un sujet historique et aux conditions singulières de ce sujet, mais la valeur d'un fait dépasse ces conditions historiques et peut être vérifié au-delà des conditions d'énonciations. Un événement, stable dans l'espace et dans le temps, peut être décrit par un grand nombre d'affirmations qui établissent des faits. Entre le monde et nos propositions sur le monde, s'installe ainsi et on pourrait tracer un fil rouge qui part de Hume et arrive jusqu'à nous en passant par Wittgenstein et Austin - un système de relations entre les choses, les événements, les individus, qui constitue notre version, notre explication du monde. Deux problèmes majeurs et bien entremêlés s'imposent ainsi : les faits nous servent-ils pour vérifier la vérité d'une affirmation? Les faits font-ils partie du monde ou sont-ils juste des descriptions? Sur ces deux questions Austin et Strawson, les deux philosophes qui, après Wittgenstein, ont bâti l'analyse du langage ordinaire, en 1950, engagent un débat autour de la notion de vérité qui reste le point de repère essentiel pour ces questions ${ }^{21}$. Pour Austin, si une affirmation (statement) est vraie, elle est vraie parce qu'elle correspond aux faits et donc, pour lui, les faits font partie de notre monde avec les objets et les événements. Selon Strawson, Austin ne distingue pas toujours les faits des événements, ce qui pose des problèmes majeurs, car «des événements peuvent être datés ou des choses localisées. Mais les faits affirmés par les affirmations (quand elles sont vraies) ne peuvent pas être ni datés ni placés [But the facts which statements (when true) state can be neither dated or located]. Sont-ils alors inclus dans le monde? $»^{22}$. Pour Strawson les faits ne sont pas des choses auxquelles les affirmations se réfèrent, mais plutôt ce que les affirmations vraies affirment (« Les faits sont ce que les affirmations assertent (lorsqu'elles sont vraies); ils ne sont pas ce à propos de quoi les affirmations disent quelque chose $»^{23}$ ).

En suivant les développements de la question, les deux positions deviennent probablement moins contradictoires à cause d'une distinction plus claire entre fait et événement et aussi grâce à l'attention portée à la nature cognitive et sociale des faits. Je fais référence, en particulier, à Searle et à sa Construction de la réalité sociale de $1995^{24}$. Les faits étudiés par Searle sont les faits institutionnels, donc des faits constitués par le langage. Mais pour les définir, Searle - en reprenant un article d'Elisabeth Anscombe de $1958^{25}$ - doit les distinguer des faits bruts, c'est-à-dire ces faits qui n'ont pas besoin des institutions pour exister.

Les baptêmes, les mariages sont des événement, mais leur validité dans le temps est donnée par certains faits : un fait brut (un morceau de papier) garde la trace d'un fait institutionnel (l'institution du mariage ou du baptême) en enregistrant un performatif (le «je te baptise», le "je te prends pour femme»). Mais l'événement individuel s'inscrit comme fait valide dans le temps aussi bien que dans l'esprit et dans la mémoire des intervenants et cette inscription n'est jamais neutre : les promesses, les espoirs, les attentes, les intentions s'inscrivent aussi dans le fait. 
La construction de notre expérience se mesure constamment avec des faits et toute compréhension des événements passe par les faits. Cela ne vaut pas seulement pour la mémoire des événements passés mais aussi pour la compréhension d'un processus, qui peut être saisi seulement à travers les traces qu'il laisse et que nous enregistrons. Mais il n'existe pas - je le répète - d'enregistrement neutre : même le fait institutionnel est déterminé - comme tout objet social - par des conditions historiques et culturelles. L'inscription (dans notre esprit ou sur un morceau de papier), l'enregistrement nous pose constamment - et bien avant la question de l'art - un problème de mise en forme, d'objectivation des faits, car de cette inscription dépend la validité de nos actes et de nos propositions. Plus rares sont, dans notre expérience, les moments où à travers des faits nous cherchons à revenir à un événement (par exemple, consulter les agendas, les cahiers, les emails pour savoir ce que j'ai fait tel jour à telle heure). Mais dans ce cas aussi, je cherche à reconstruire et à situer dans l'espace et dans le temps un épisode sans nécessairement m'interroger sur le sens de l'événement.

Mais quel est le rapport entre faits et documents et entre événements et monuments ? Et comment la coexistence de ces deux polarités peut caractériser une œuvre d'art?

\section{The time capsule}

Continuons alors par une petite promenade à New York. Au Flashing Meadows Park, dans le Queens, il y a un cylindre de granite de sept tonnes, surnommé « the permanent sentinel » (FIG. 01). Comme nous l'indique l'inscription (FIG. 02), à quinze mètres sous ce bloc deux time capsules (la première de 1938, la deuxième de 1965) ont été enterrées par la Westinghouse Electric Corporation, à l'occasion des deux éditions de la New York World's Fair correspondantes. Une time capsule est tout simplement un contenant conçu pour résister au temps et préserver des documents - principalement d'une culture matérielle - pour les époques à venir. Dans ce sens, Pompéi par exemple peut être considérée comme une énorme time capsule involontaire. Les deux time capsules de la Westinghouse Corporation sont au contraire intentionnelles et, bien qu'elles soient les plus célèbres, elles ne sont pas les seules. L'International Time Capsule Society, de l'Oglethorpe University d'Atlanta, a estimé qu'il existe dans le monde environ 15000 time capsules, dont quatre-vingt pour cent sont désormais perdues, ce qui permet d'envisager des time capsules comme ruines. 
Figure 1 :

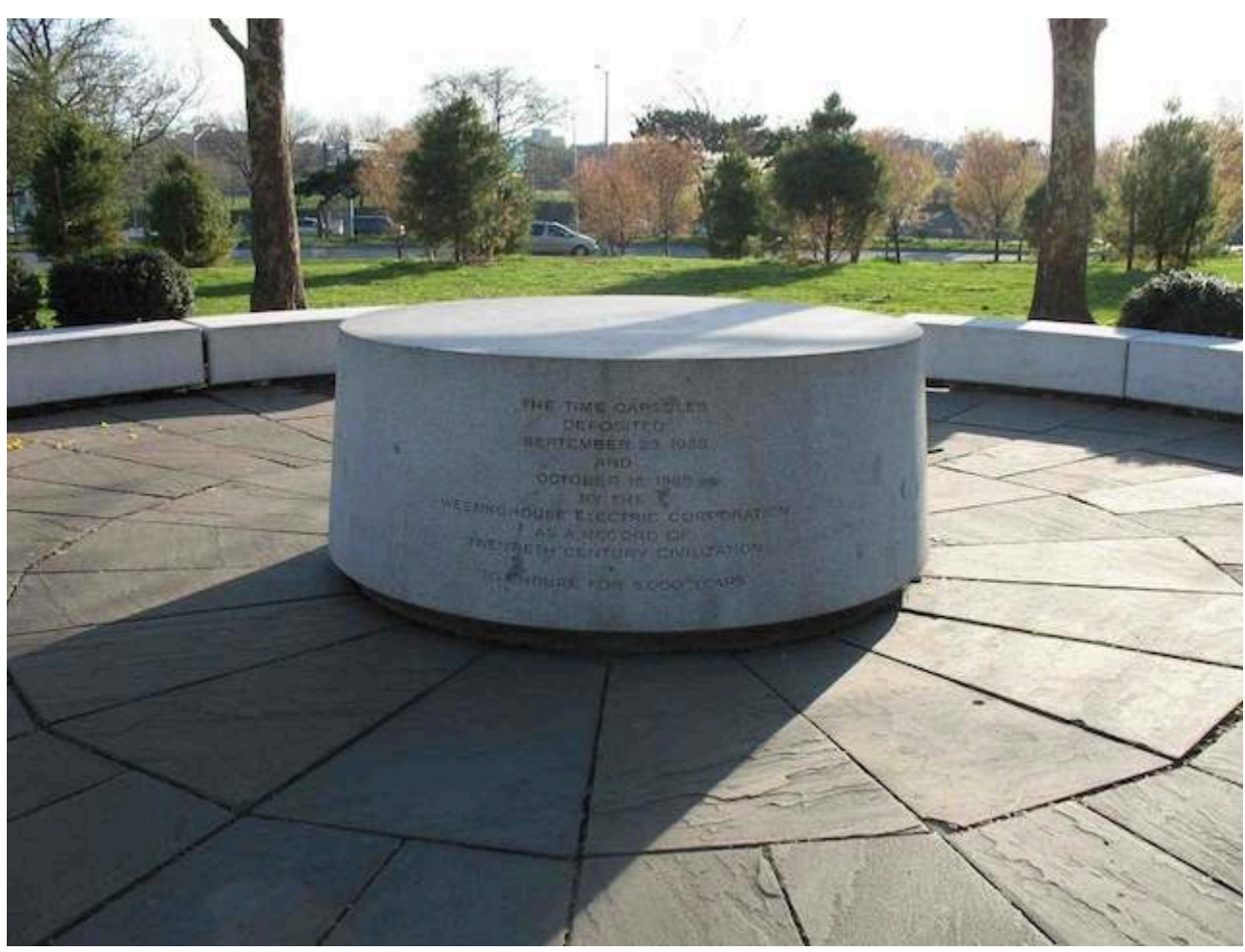

Time Capsules Monument, Flashing Meadows Park, New York.

\section{Figure 2 :}
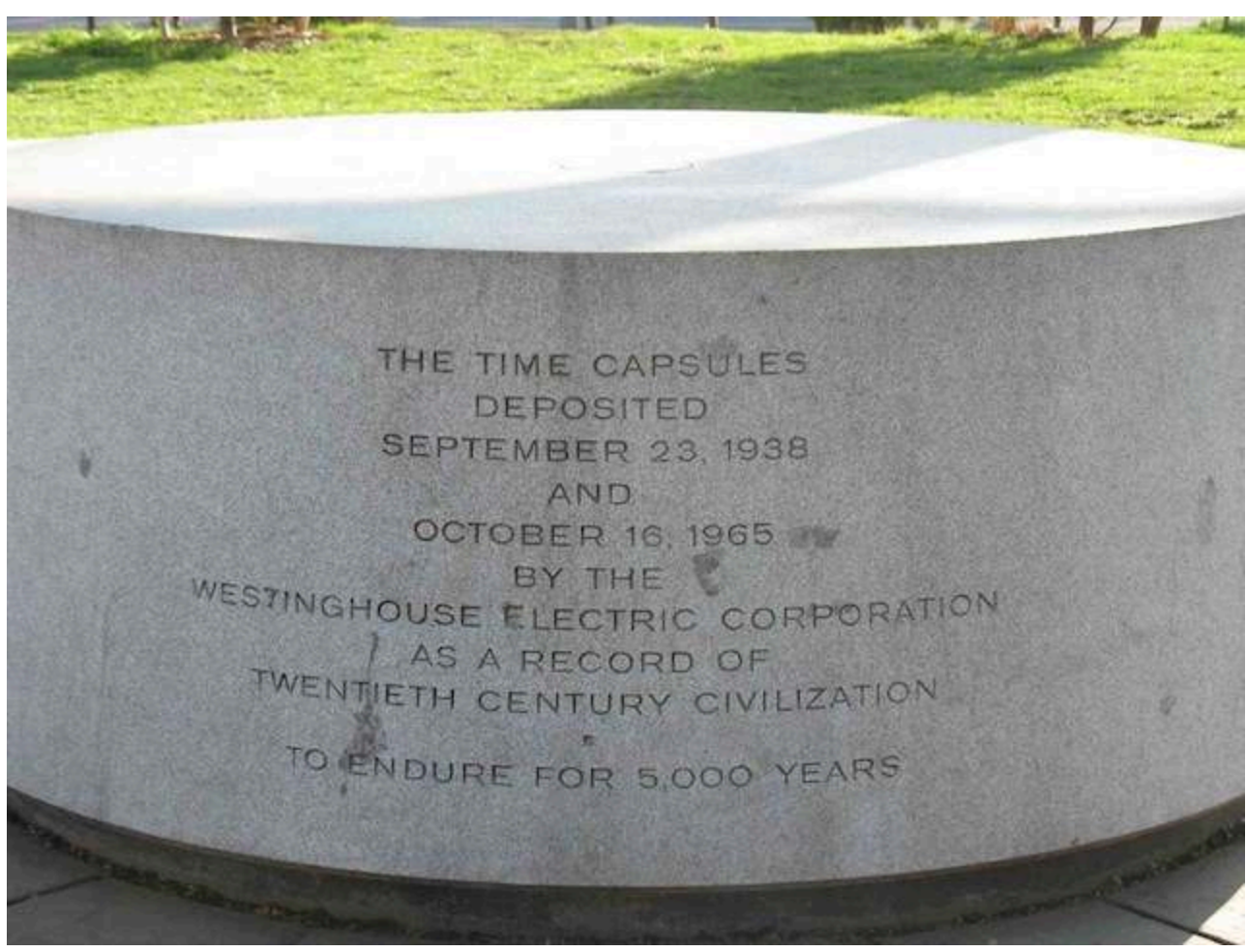

Time Capsules Monument, Flashing Meadows Park, New York. 
19 Pour l'instant, celle de 1938 (FIG. 03) nous suffit largement pour imaginer les pauvres humains d'un futur en ruines (FIG. 04). Il s'agit d'une sorte de fusée construite avec un alliage métallique spécial, capable de résister 5000 ans et destinée à donner aux hommes de 6938 une "représentation" de 1938. À son intérieur, se trouvent les objets les plus variés : des lunettes, une ampoule, un stylo, un appareil photo, un paquet de cigarettes Camel, des échantillons de tissu et de métal et des microfilms de livres, de revues, de pages d'encyclopédies, d'œuvres d'art, de romans.

Figure 3 :

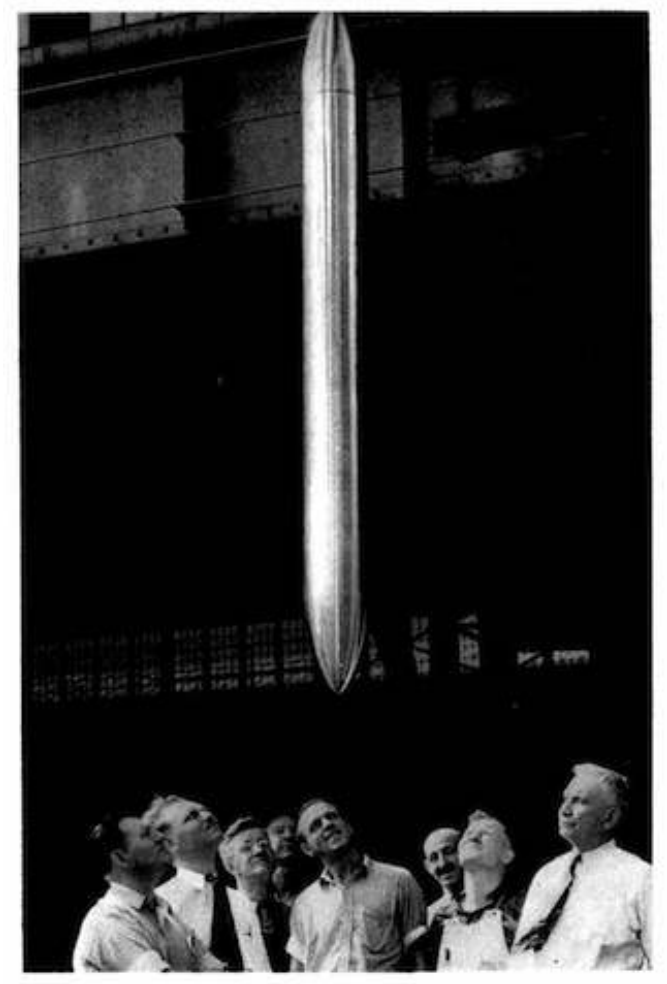

Image de la Time Capsule de 1938 in The Book of Record of the Time Capsule of Cupaloy, Deemed Capable of Resisting the Effects of Time for Five Thousand Years, Preserving an Account of Universal Achievements, Embedded in the Grounds of the New York World's Fair, 1939, New York, Westinghouse, 1938 
Figure 4 :

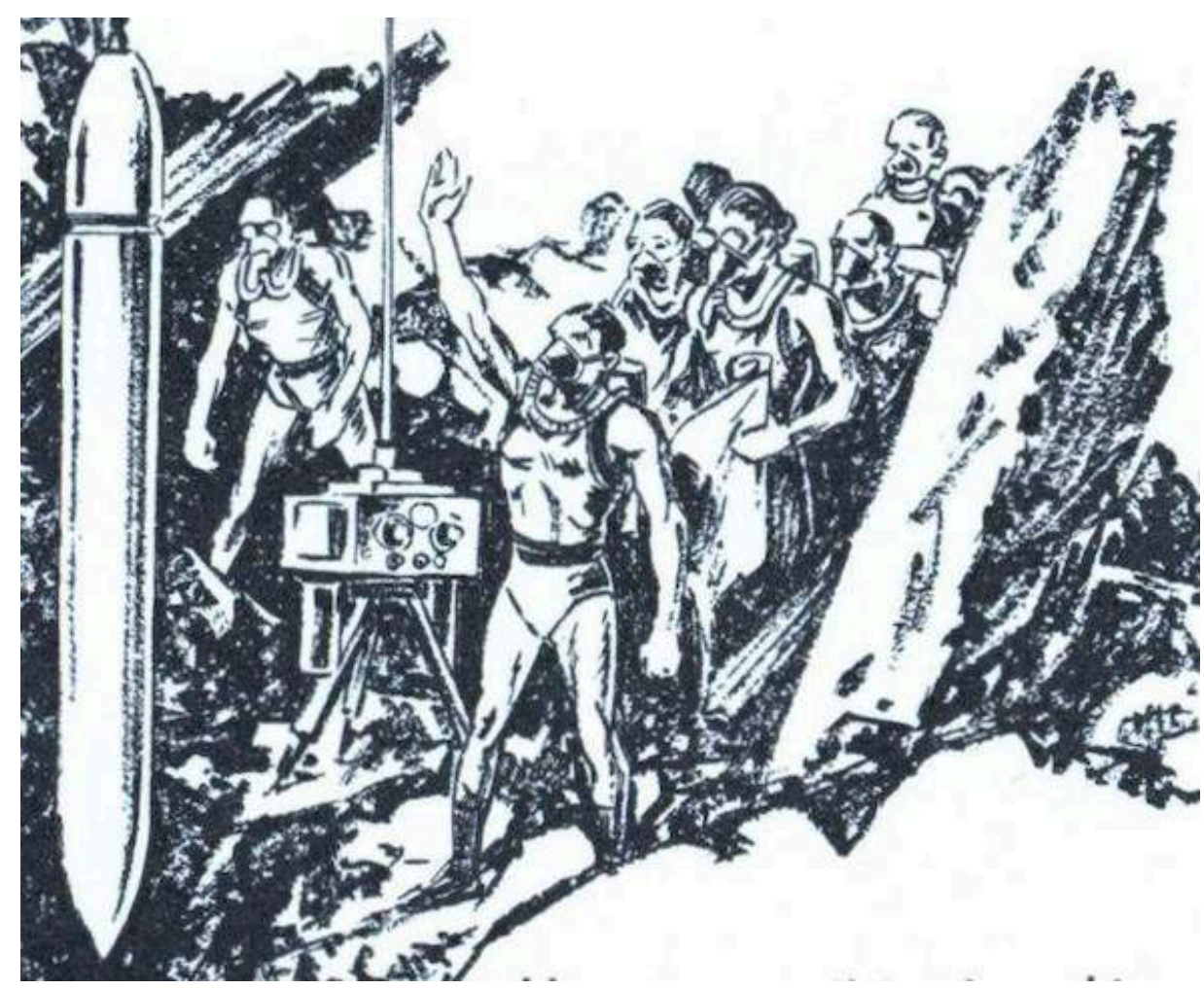

Illustration humoristique de la time capsule (source inconnue).

Deux publications, distribuées en plusieurs milliers d'exemplaires, accompagnent l'événement: The Story of the Time Capsule et The Book of Records. Toutes les deux sont pour moi du plus grand intérêt, car la première décrit le processus technique et idéologique qui a conduit à la réalisation de ce monument au progrès et à l'entreprise américaine et nous présente la liste des documents contenus dans la capsule, alors que la deuxième offre, en quelque sorte, le mode d'emploi (FIG. 05 et FIG. 06). 
Figure 5 :

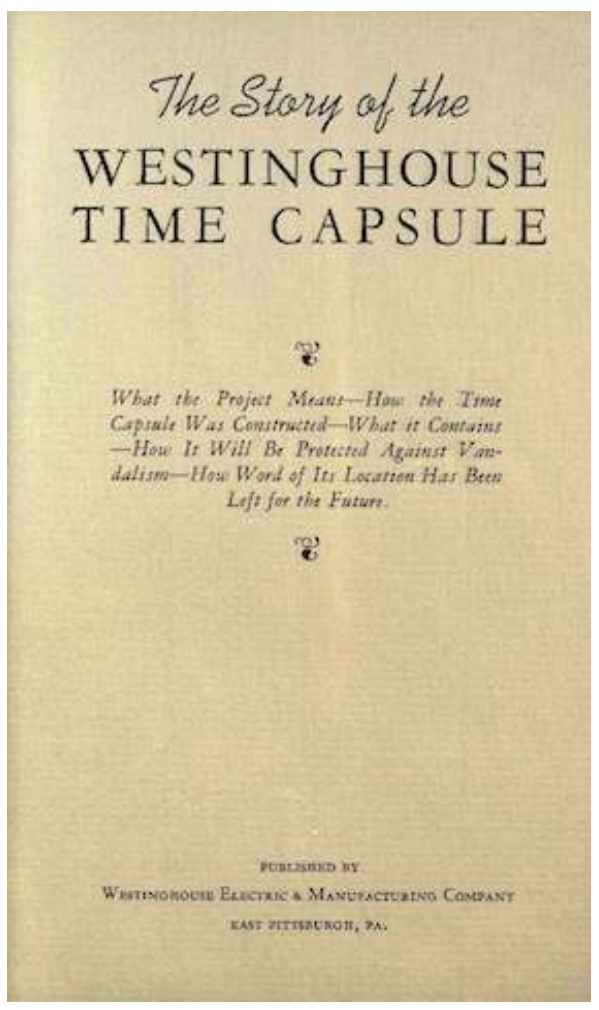

The Story of Time Capsule, New York, Westinghouse, 1938.

\section{Figure 6 :}

THE BOOK OF RECORD OF

\section{THE}

\section{TIME CAPSULE}

\section{OF CUPALOY}

DEEMED CAPABLE OF RESISTTNG

THE EFFECTS OF TIME FOR FIVE THOUSAND YEARS - PRESERVING

AN ACCOUNT OF UNIVERSAL ACHIEVEMENTS - EMBEDDED IN THE GROUNDS OE THE

NEW YORK WORLDS FAIR 1939

SEPTEMBER 23.1938

The Book of Record of the Time Capsule of Cupaloy, New York, Westinghouse, 1938. 

mais aussi le ton de l'entreprise : «Depuis toujours les archéologues et les historiens ont consacré leurs talents à déchiffrer le passé sans documents (unrecorded past), et les êtres humains ont rêvé de simplifier le problème pour les savants futurs, en préparant de manière délibérée un message de notre temps pour eux. Jusqu'à aujourd'hui ce rêve perpétuel n'a été qu'un rêve ». Il nous présente ensuite l'effort technique qu'il a fallu fournir pour créer cette fusée résistante au temps. The Book of Records, imprimé avec une encre spéciale sur un papier spécial est, avec la Bible, le seul livre contenu dans la time capsule. Il offre des instructions pour déchiffrer ce « segment de notre temps pour les générations futures ", un précis de langue anglaise ainsi que trois messages: le premier du Prix Nobel de physique Millikan, le deuxième de Thomas Mann et le dernier d'Einstein. Voici justement le message de Mann : «Nous savons maintenant que l'idée $d u$ futur comme un "monde meilleur" a été une erreur de la doctrine du progrès. Les espoirs que nous portons sur vous, citoyens de demain, ne sont en aucune façon exagérés. (...) Cette conception optimiste de l'avenir est une projection dans le temps d'une détermination qui ne fait pas partie du monde temporel: l'effort de la part de l'homme de se rapprocher de son idée de lui-même, l'humanisation de l'homme. Ce que nous, en 1938, comprenons par le terme "culture", une notion peu estimée aujourd'hui par certaines nations du monde occidental, est tout simplement cette détermination. Ce que nous appelons l'esprit coïncide aussi avec elle. Frères de l'avenir, unis avec nous dans l'esprit et dans cette détermination, nous vous envoyons nos salutations ». La construction des documents et des monuments correspond justement à cette volonté d'humanisation.

Dans notre hypothèse, la time capsule peut être considérée comme exemplaire pour deux raisons. La première est qu'elle offre une très bonne illustration de la construction à la fois du document et du monument, en exemplifiant de façon assez claire le rapport - et la différence - entre document et monument.

La deuxième raison est tout simplement que la time capsule n'est pas une œuvre d'art, même si elle présente certains "symptômes" qu'on pourrait rattacher à une œuvre d'art.

Ces deux raisons ne sont pas évidentes et nécessitent cependant quelques éclaircissements.

\section{Deux questions sur le document et le monument}

Qu'est-ce qui donne aux objets contenus dans la time capsule le statut de documents? Et dans quels sens pouvons-nous parler du permanent sentinel comme d'un monument?

Le document est un objet social dont l'existence dépend d'un acte d'inscription ${ }^{26}$. Donc, en revenant à notre time capsule, une ampoule, un paquet de cigarettes sont des documents parce qu'un livre liste ces documents et explique l'intention de la liste. Les exemplaires de ce livre sont eux-mêmes des documents car ils sont à leur tour inscrits dans des catalogues. Sans archives, sans catalogues, sans registres, sans actes, il n'y aurait pas de documents. Bref, ils sont des documents à partir du moment où ils intègrent une culture écrite.

Dans un célèbre article paru dans l'Enciclopedia Einaudi et ayant pour titre Document/ Monument, Jacques Le Goff écrit: «La mémoire collective et sa forme scientifique,

Images Re-vues, 11 | 2013 
l'histoire, s'appliquent à deux types de matériaux : les documents et les monuments. [...] Les monuments, héritage du passé, et les documents, choisis par l'historien ${ }^{27}$. Cette distinction peut renvoyer à l'essai de 1903, Le culte moderne des monuments ${ }^{28}$, où Riegl distinguait les monuments intentionnels des monuments historiques ou involontaires. L'essai de Le Goff trace ensuite l'évolution de l'idée de document dans les sciences historiques, jusqu'à arriver, à travers ce qu'il appelle la révolution documentaire, à la notion contemporaine de document défini par une polarisation avec le monument, au point de penser le document/monument comme un concept en soi : « Le document n'est jamais innocent. Il est le résultat avant tout d'un montage, conscient ou inconscient, de l'histoire, de la société qui l'a produit, mais aussi des époques successives pendant lesquelles il a continué à vivre, peut-être oublié, pendant lesquelles il a continué à être manipulé, peut-être en silence. Le document est une chose qui reste, qui dure et le témoignage, l'enseignement qu'il porte doivent être avant tout analysés en démystifiant leur signification apparente. le document est monument. Il est le résultat de l'effort des sociétés historiques pour imposer - volontairement ou involontairement - telle image d'elles-mêmes au futur. Il n'y a pas, à la limite de document-vérité. Tout document est mensonge. Il appartient à l'historien [...] en premier lieu [de] démonter, démolir ce montage, déstructurer cette construction, et d'analyser les conditions de production des documents-monuments $»^{29}$. À la limite, ajoutons-nous, il n'existe pas de monument involontaire, c'est-à-dire de monument qui ne soit pas l'expression d'une intentionnalité. Tant le document que le monument sont des constructions, des mises en forme du réel, qui introduisent deux directions temporelles divergentes, celles $d u$ fait et de l'événement. Chaque document est déterminé par une culture historique individuelle ou collective : chaque acte de documentation est destiné à devenir un monument intentionnel pour les générations futures.

Or - et je reviens à la deuxième question que je posais tout à l'heure - quel genre de monument est ce permanent sentinel ? Est-ce qu'il s'agit vraiment d'un monument ? Oui, même si son rapport à la mémoire est assez particulier. Il s'agit bien sûr d'un genre de monument intentionnel, à la fois plaque commémorative et pierre tombale. Ces deux types de monument ont un point en commun important pour une définition restreinte du monument: l'ici. Lorsque la validité du document en tant que fait enregistré est indépendante de sa collocation spatio-temporelle, la valeur d'un monument est strictement dépendante d'un lieu: on peut même dire qu'un monument constitue un lieu, destine un lieu à une fonction de remémoration. Pour remplir cette fonction, un monument a besoin de sujets qui ont inscrit dans leur mémoire l'événement ou la personne dont le monument est monument. On peut aussi ajouter qu'un monument a besoin alors, pour garder sa fonction, de documents qui préservent la mémoire. Sans cette polarité entre le monument et les documents, nous serions face à une ruine. Mais lorsque les documents (leur préservation, leur construction) déterminent la mémoire en termes d'information, de version, d'archive - bref, de faits de mémoire - le monument est censé constituer un événement de mémoire, c'est-à-dire non pas la représentation d'un événement du passé, mais l'acte de remémoration en soi : l'acte de remémoration comme actualisation de l'événement.

Le permanent sentinel, dans sa simplicité, est donc la construction d'un événement bien précis et bien localisé dans l'espace et dans le temps : un rendez-vous ici au Flashing Meadows Park, en 6939. On pourrait se demander comment, au moment de ce rendez- 
vous, les hommes du futur traiteront les documents de la time capsule. Comme des monuments, peut-être, ou bien comme réactualisations d'événements.

\section{Pourquoi la time capsule - dans son ensemble - n'est-elle pas une œuvre d'art?}

30 En toute honnêteté, je pense qu'il ne s'agit pas d'une question particulièrement intéressante et personne, je crois, n'a jamais éprouvé le besoin de la poser. Mais, ex negativo, cette question peut nous donner quelques éléments de compréhension. Nous pouvons indiquer au moins quatre raisons pour lesquelles la time capsule n'est pas une œuvre d'art. Les deux premières sont assez évidentes et directement liées aux intentions et au contexte : cet objet n'est pas né pour répondre à une intention d'art, ne demande pas à être reconnu, regardé, évalué comme œuvre d'art et il a été présenté dans un contexte et à un public qui n'ont rien à voir avec une quelque idée d'art. Bref, rien ne nous indique qu'il s'agit d'une œuvre d'art. En d'autres termes, si une œuvre d'art est aussi un objet culturel, le système culturel d'origine ne pouvait pas intégrer cet objet dans une théorie de l'art (le système culturel d'aujourd'hui, peut-être). La time capsule n'agit pas comme une œuvre d'art, même si nous pouvons y reconnaître quelques symptômes artistiques : l'eccéité n'est pas la quiddité et il sera bien difficile de retrouver là un type culturellement déterminé. Ces deux raisons sont bien évidemment des raisons historiques. Mais je voudrais suggérer aussi deux raisons métahistoriques. Le premier est que la finalité de cet objet est déclarée et même inscrite sur la fusée et dans le mode d'emploi. L'homme du futur qui l'ouvrira est censé - au moins dans les intentions des hommes de la Westinghouse Corporation - comprendre la finalité de l'objet et le traiter selon ces finalités. Et d'ailleurs ces finalités sont externes à l'objet en soi. La deuxième raison nous mène finalement au rapport entre document et monument : quinze mètres de terre et cinq mille ans de distance entre les deux : pas de polarité, pas de coexistence, pas de tension. Les faits (les documents) restent bien distingués des événements (le monument) comme dans l'ordinaire.

\section{Document/monument : deux exemples de polarisation}

31 Deux exemples artistiques peuvent nous montrer une articulation forte de la polarité entre document et monument, entre fait et événement, basée sur leur coexistence. Il s'agit des œuvres de deux artistes, Richard Serra et Walker Evans, qui ont eu en commun une réception critique parfois controversée : les œuvres du premier ont été souvent étiquetées comme monumentales, celles du deuxième comme documentaires. Et les deux artistes ont mal réagi à ces étiquettes. Voici un entretien de 1980 de Serra avec Douglas Crimp. «Douglas Crimp: "Avant de parler des deux sculptures du sud de Manhattan [il s'agit du St John's Rotary Arc FIG. 7 et de TWU] tu peux peut-être me faire un rapide historique de tes travaux antérieurs en milieu urbain, ce que l'on pourrait appeler ta sculpture publique ou monumentale [FIG. 8]." Richard Serra: "Je ne veux pas parler de la question de la sculpture monumentale. Je n'adhère pas à ce concept. Je ne vois pas mon travail comme monumental $»^{30}$. 
Figure 7:

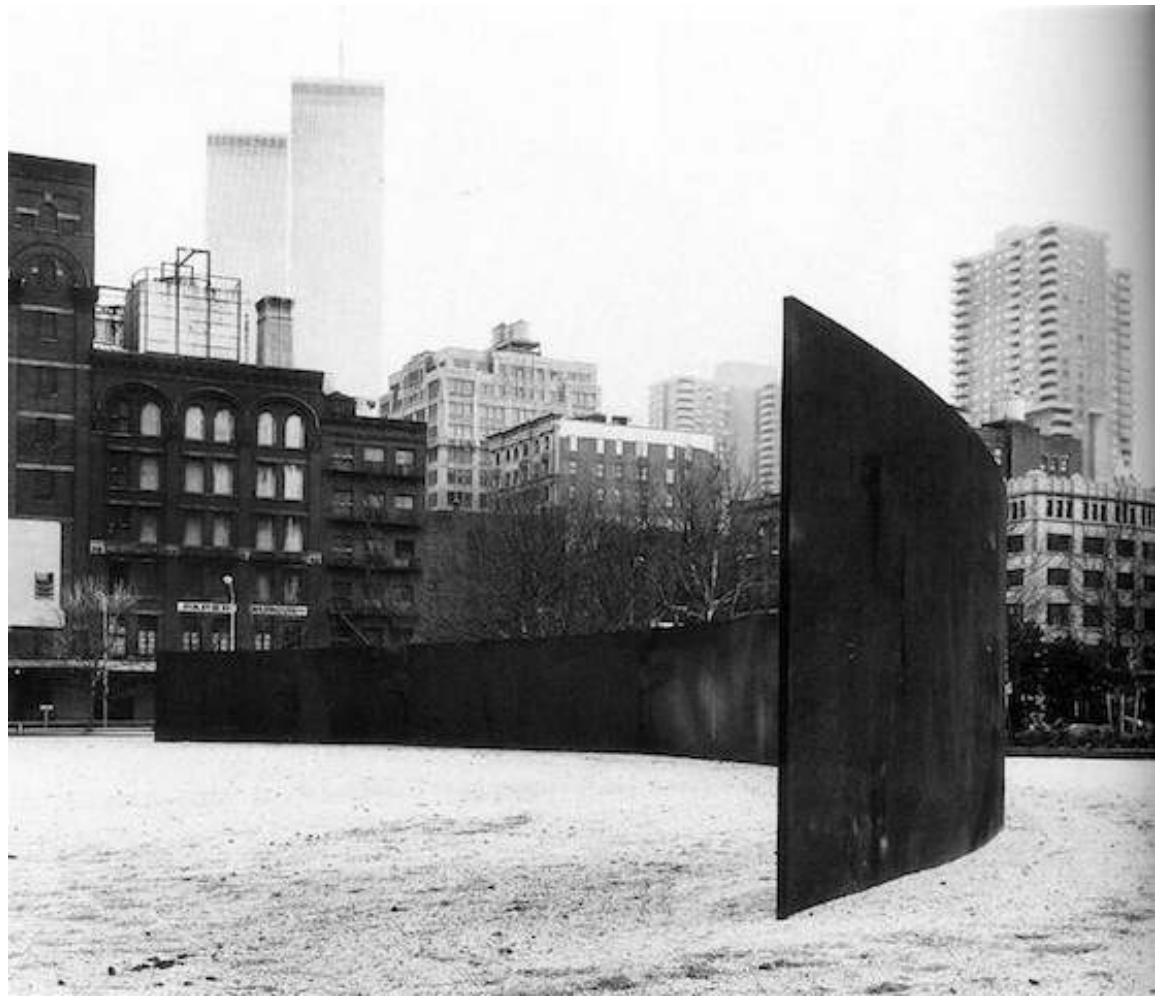

Richard Serra, St John's Rotary Arc, 1975/80, Holland Tunnel Exit, New York.

Figure 8:

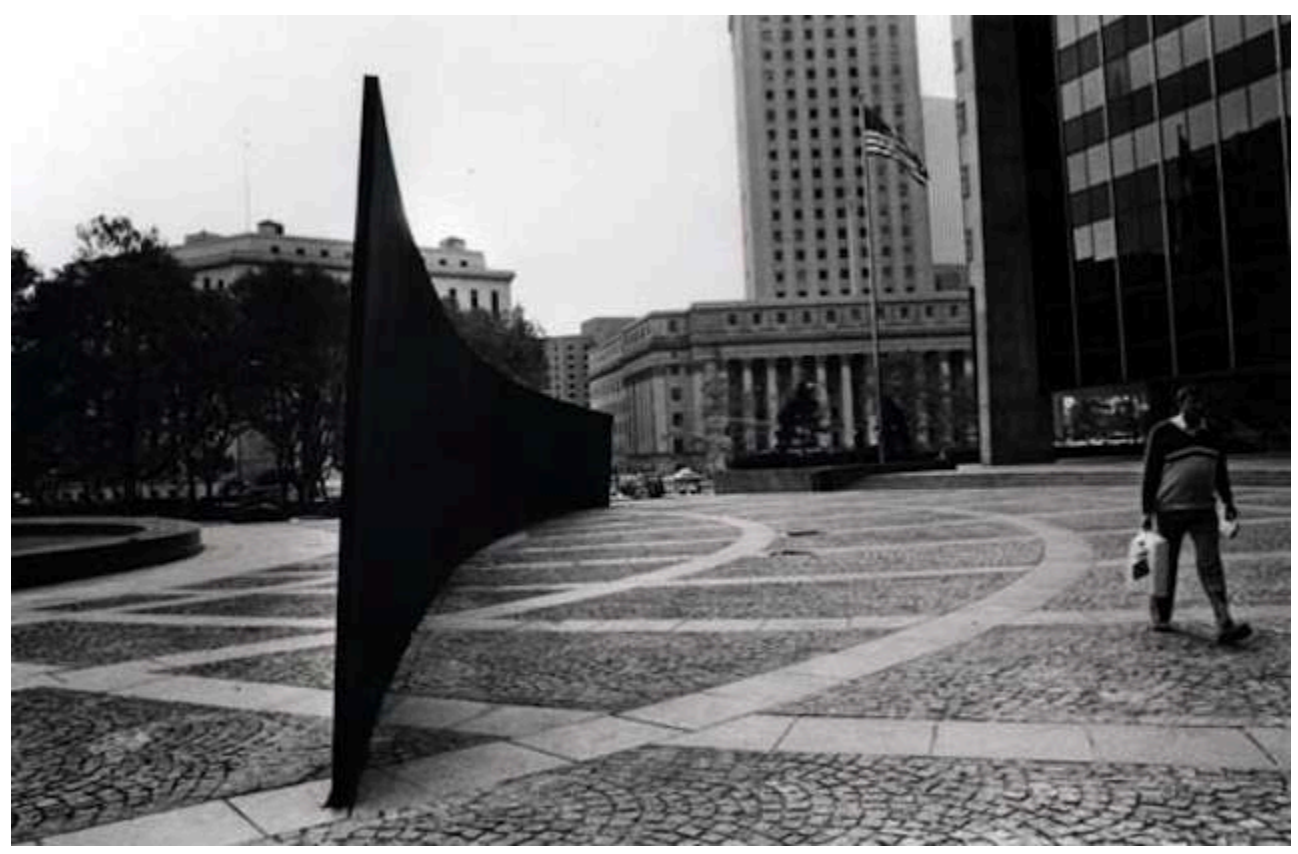

Tilted Arc, Richard Serra, 1981, sculpture, steel, New York City (destroyed). Photo (c) 1985 David Aschkenas.

Ce que Serra veut probablement dire est que ses œuvres ne sont pas le souvenir de quelque chose, n'ont pas une dimension commémorative, ne sont pas à-propos-d'un 
événement du passé. Bref, il refuse pour celles-ci l'aspect de narration, de figuration, de représentation du monument. Ces œuvres sont des faits qui interviennent dans le monde-de-la-vie, pour construire une version du monde. Mais en même temps elles ne sont pas réductibles aux documents, comme le montre bien une remarque de Serra à propos des photographies des sculptures. Serra déclare : «Si on réduit la sculpture au plan, on ne fait passer qu'une sorte de résidu de ses préoccupations. On nie l'expérience temporelle de l'œuvre. Non seulement on la réduit à une échelle qui n'est pas la sienne, pour des raisons de consommation, mais on nie le contenu réel de l'œuvre. Toujours est-il que pour la plupart des sculptures, on ne peut pas faire l'expérience de l'œuvre si on la sépare de son emplacement propre. En dehors de cette condition, toute expérience de l'œuvre est une tromperie $~{ }^{31}$. Au contraire, l'œuvre est l'expérience, l'œuvre est la construction des conditions d'une expérience. Et sans cette expérience l'œuvre n'a plus de contenu. Et à la fois, l'œuvre est un fait installé dans le monde-de-lavie, à la fois monde de l'évidence et de l'énigme, le monde où les hommes agissent en relation mutuelle, où on découvre, déjà dans la perception quotidienne des choses, le sentiment intersubjectif.

L'œuvre nous rappelle que l'événement - cet événement - doit rester soudé au mondede-la-vie. C'est un événement perceptif, où percevoir signifie aussi se souvenir, sentir, comprendre à travers une histoire et à travers nos sens. L'art nous rappelle que toute construction des faits, toute construction du monde, revient à l'expérience du sujet, sans laquelle il perd son sens. Serra refuse l'étiquette de monument pour ses œuvres parce qu'il veut revenir aux faits, c'est-à-dire aux constructions singulières, aux descriptions subjectives de l'expérience. En d'autres termes, les expériences sont toujours construites et il n'y a jamais des événements purs comme il n'y a pas de donnés purs : au contraire, la construction des faits est - dans l'espace esthétique proposé par une œuvre - la condition même de tout événement. Les œuvres de Serra ne sont ni des monuments (monuments à...) ni, bien sûr, des documents (documents de...). Elles nous invitent donc à voir le couple document/monument comme une facette du couple fait/événement.

Evans a eu un destin parallèle à celui de Serra, bien que diamétralement opposé. Son travail a été continuellement désigné comme documentaire. Dans un entretien de 1971, Walker Evans répondait ainsi à une question sur le rapport entre photographie documentaire et photographie d'art : « Documentaire ? C'est un mot très sophistiqué et trompeur. Et ce n'est pas vraiment clair. Vous devez avoir une oreille sophistiquée pour recevoir ce mot. Le terme doit être style documentaire. Un exemple littéral de document serait une photographie policière d'une scène de crime. Vous voyez, un document est employé, alors que l'art est totalement inutile. C'est pourquoi l'art n'est jamais un document, bien qu'on puisse certainement adopter ce style ${ }^{32}$. Dans le même entretien, Lesle Katz, l'interlocuteur d'Evans, commentait: «En littérature ou en peinture, l'artiste crée dans une prolongation du temps. La photographie est un instant. Vos photos montrent la monumentalité de l'instant $\aleph^{33}$. L'acte d'objectivation propre au style documentaire, l'isolement de la figure ou de l'objet, produit en effet, dans les œuvres d'Evans, une monumentalisation de l'objet même, en montrant de manière assez efficace la polarité entre document et monument. Les photos d'immeubles d'Evans (FIGG. 9, 10 et 11) possèdent, à mon avis, ce caractère monumental et nous permettent d'explorer un petit peu la différence temporelle entre le fait et l'événement. La monumentalisation de l'instant peut être ici considérée comme 
extraction, abstraction d'un instant de la durée et à la fois comme la possibilité de répéter cet instant, de renouveler son caractère d'événement. Au contraire, le document est juste une trace, un indice, du passage de l'événement. Alors, pour reprendre les mots d'Evans, on peut avoir un style documentaire, c'est-à-dire une manière de composer l'image qui rappelle les photos de la police. Mais les faits seuls ne peuvent pas satisfaire l'expérience esthétique. Il faut revenir à l'instant, au monument, à l'événement. Mais comment y arriver sans une construction des faits?

\section{Figure 9 :}

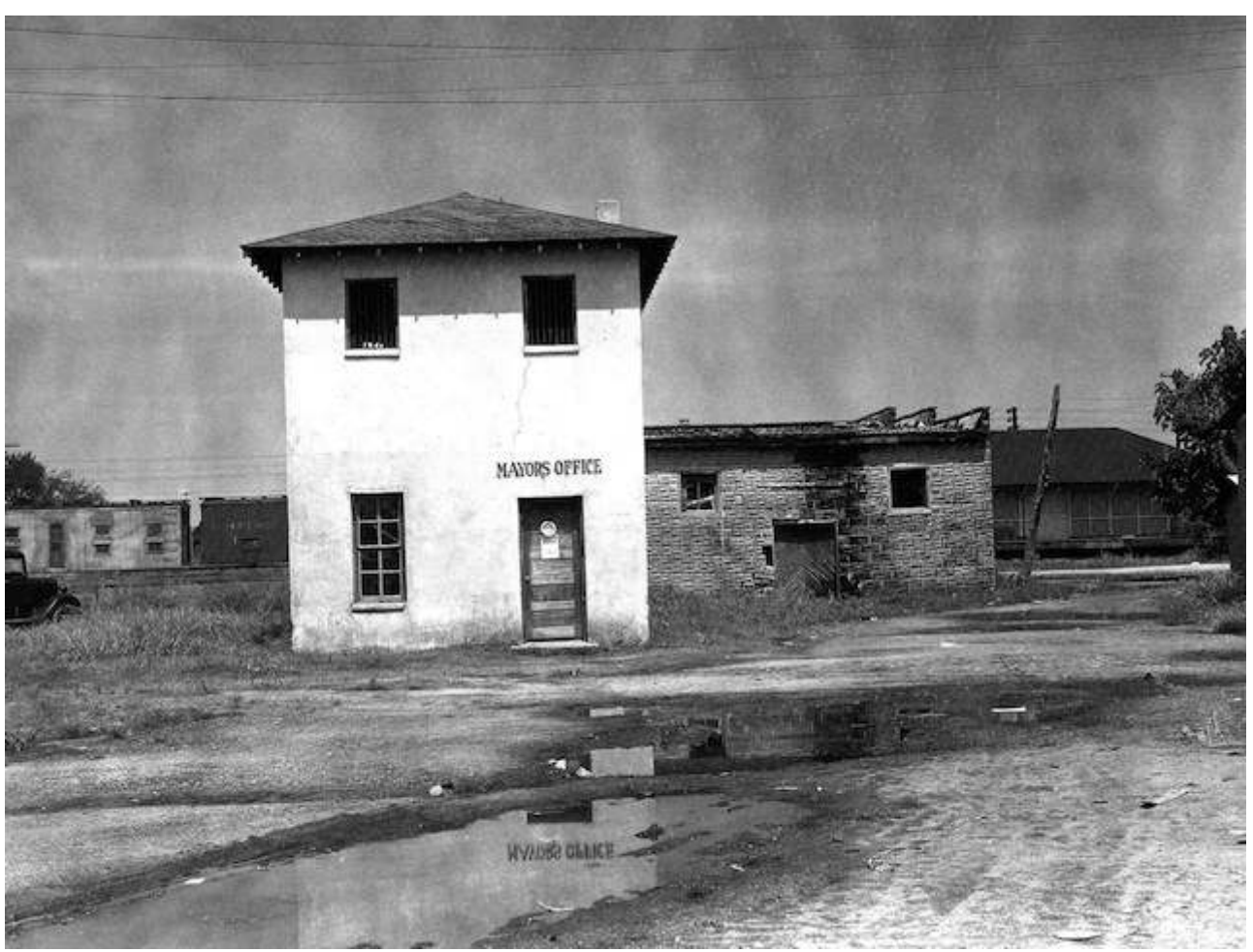

Walker Evans, Mayors House, 1936 
Figure 10 :

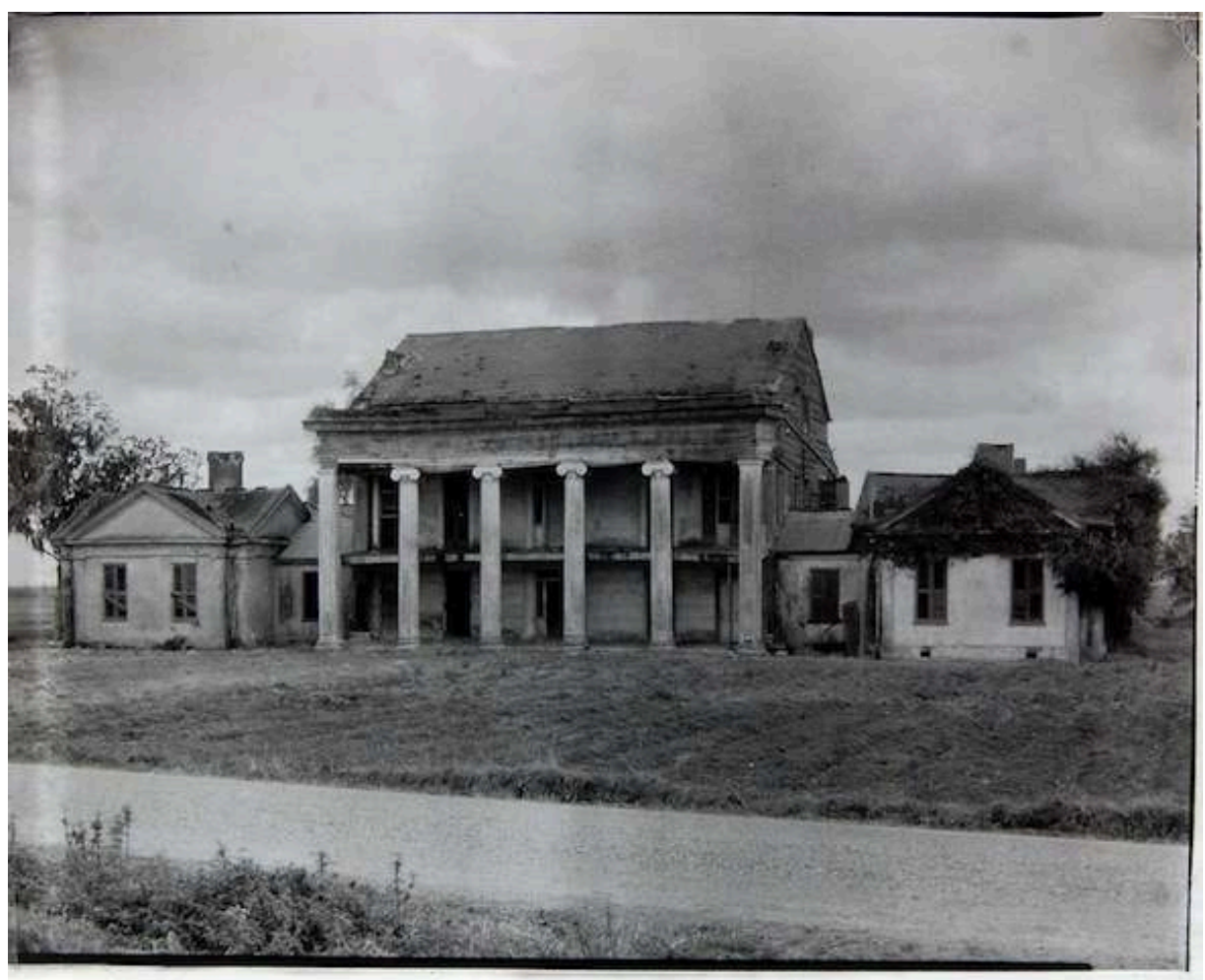

Walker Evans, Woodlawn Plantation, Belle Chase, Louisiana, 1935

Figure 11:

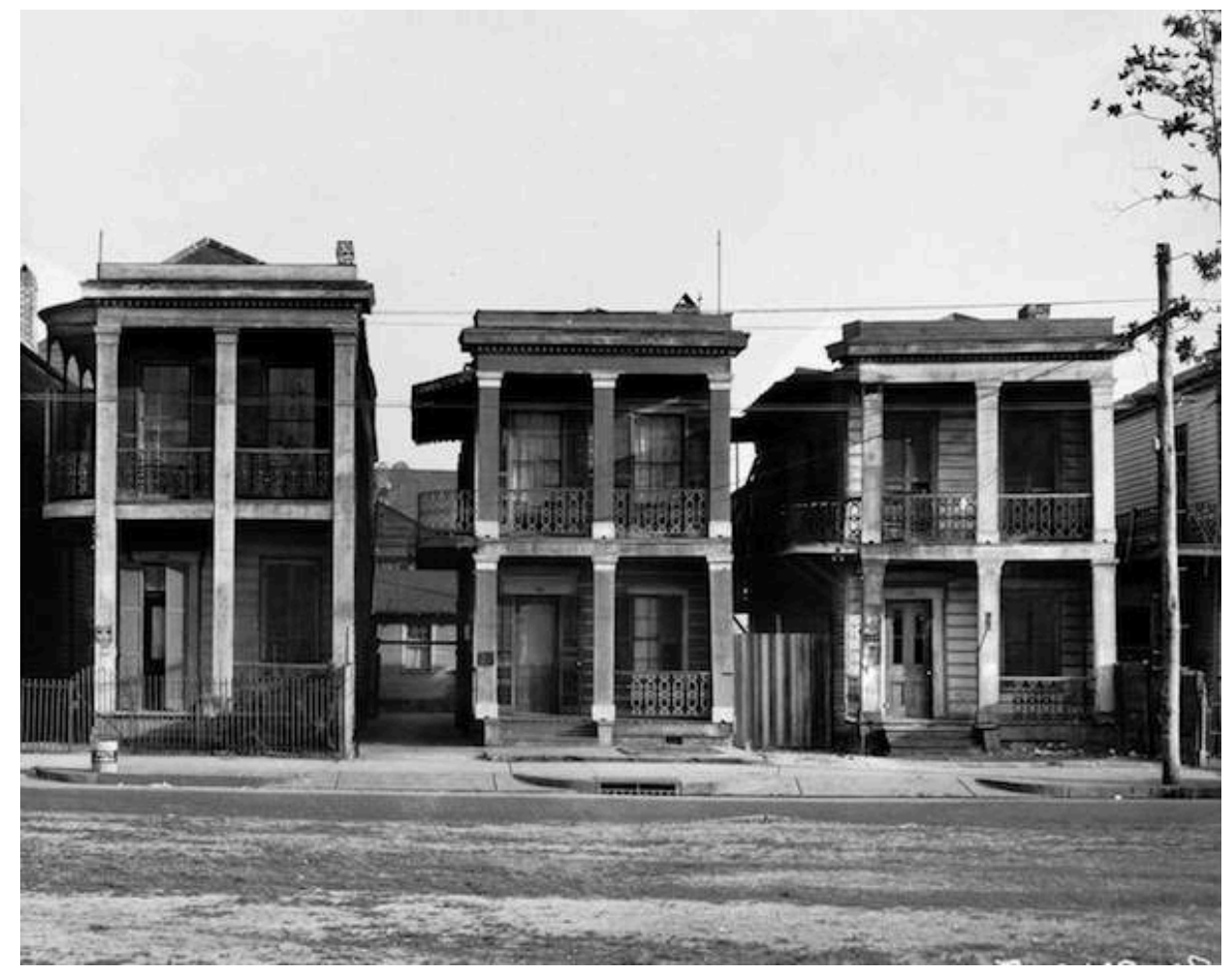

Walker Evans, New Orleans Houses, 1935. 


\section{Conclusions} passera pour oublier ce monument ou pour le redécouvrir, n'effacent pas la tension entre documents et monument, entre les milliers de faits singuliers inscrits sur le plomb et l'événement de leur mise en relation. 
Figure 12:

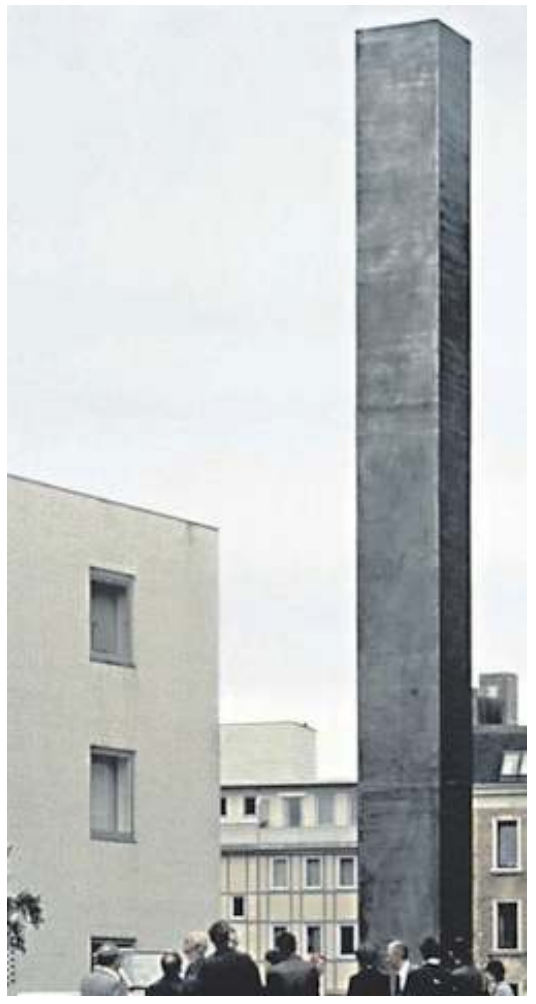

Jochen Gerz, Esther Shalev Gerz, Monument contre le Fascisme, 1986-1993, Harbourg 1986.

Figure 13:

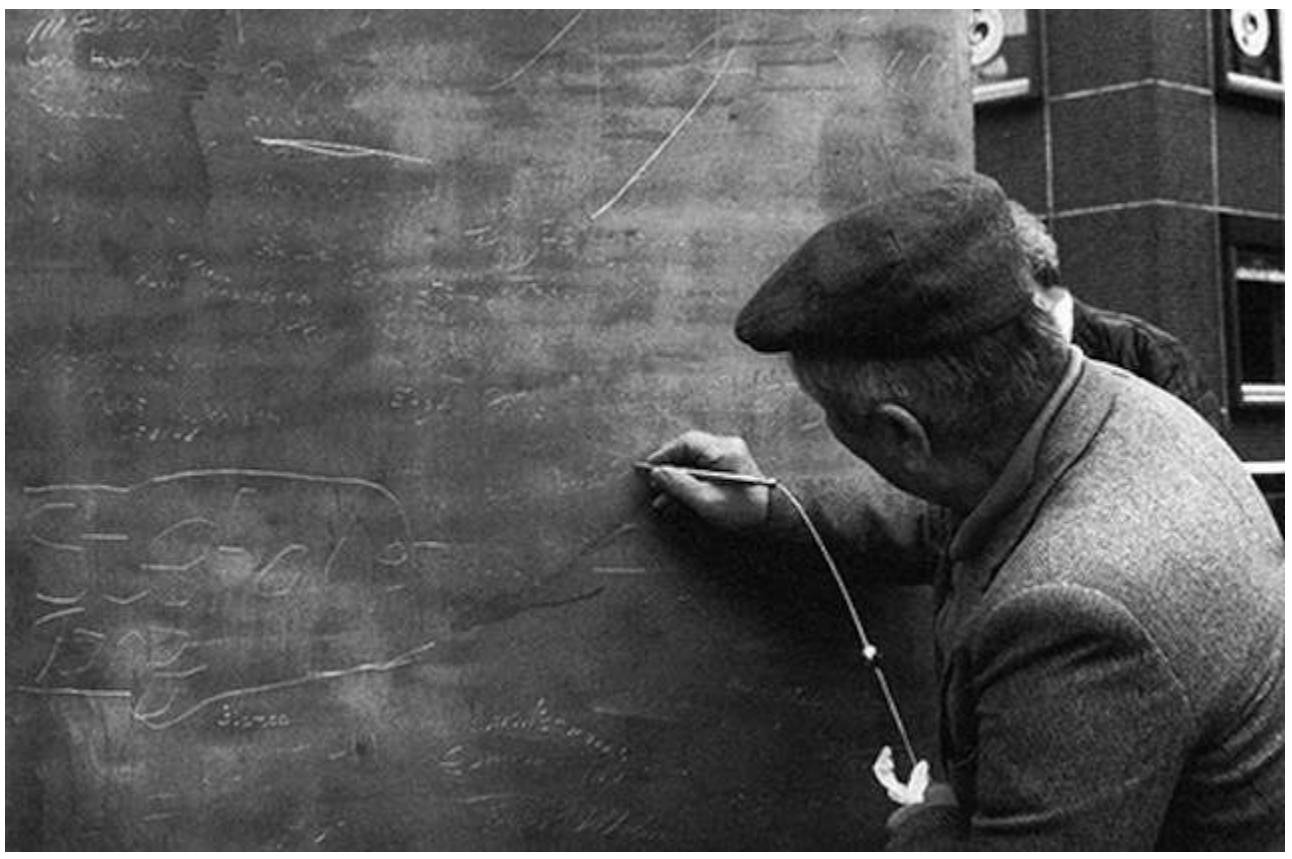

Jochen Gerz, Esther Shalev Gerz, Monument contre le Fascisme, 1986-1993. Homme signant le monument, image disponible sur http://blog.sfmoma.org/2012/07/a-meditation-on-space-in-fourparts/ 
Figure 14 :

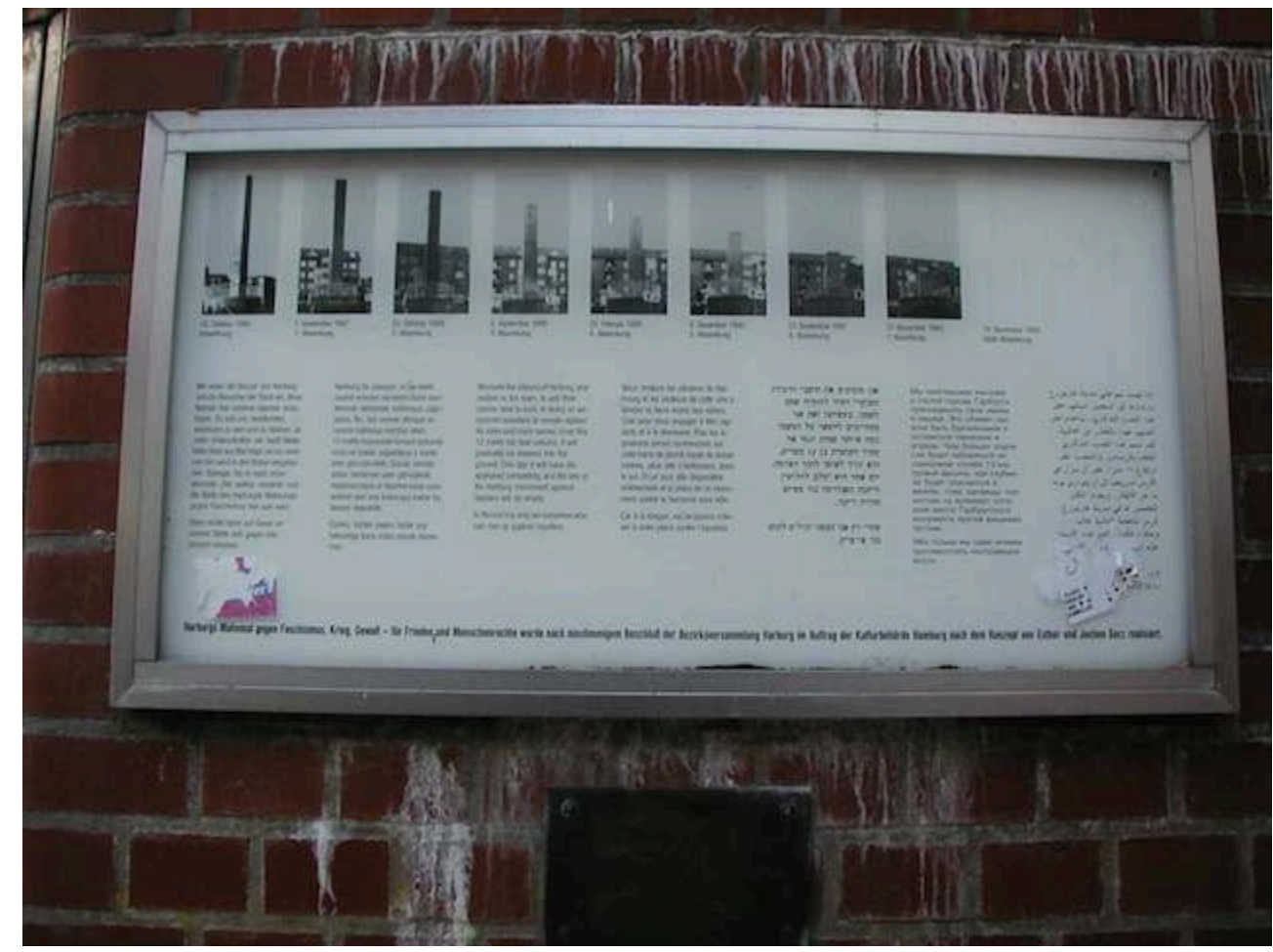

Jochen Gerz, Esther Shalev Gerz, Monument contre le Fascisme, 1986-1993. Tableau dans le quartier Harburg montrant la construction du monument.

Figure 15:

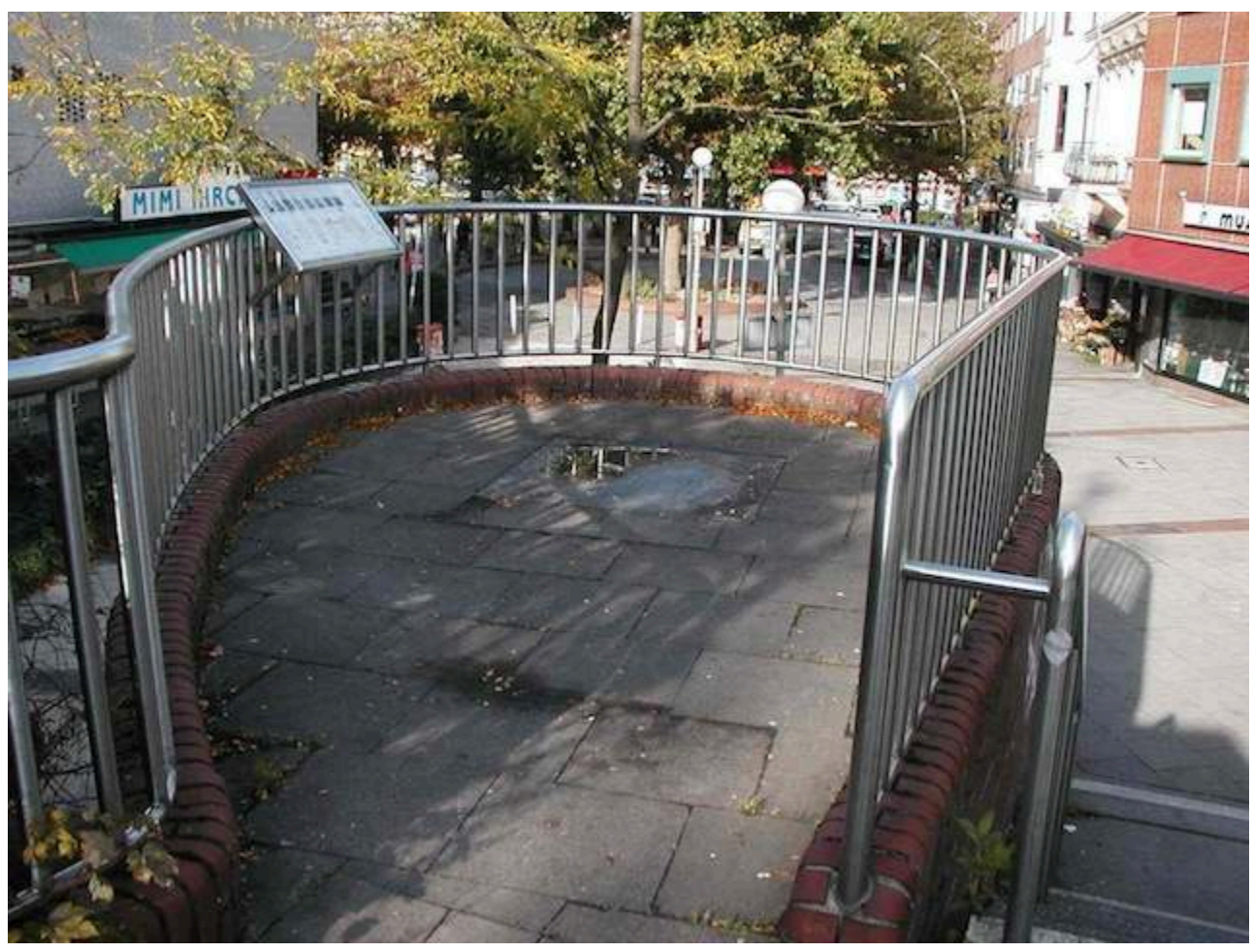

Jochen Gerz, Esther Shalev Gerz, Monument contre le Fascisme, 1986-1993. Le mémorial après 1993. 


\section{NOTES}

1. Je tiens ici à remercier Bénédicte Duvernay, Nadia Fartas et Philippe Rousseau pour leurs relectures attentives. Une première version de ce travail avait été présentée le 11 décembre 2010 dans le cadre du séminaire dirigé par Danièle Cohn, Spatialisation de la mémoire du CEPA (Culture, Esthétique et Philosophie de l'Art) - Paris 1 Panthéon-Sorbonne. Je remercie ici Danièle Cohn et les participants au séminaire dont les suggestions m'ont permis de préciser la direction de cette recherche.

2. E. Panofsky, Über das Verhältnis der Kunstgeschichte zur Kunsttheorie. Ein Beitrag zu der Erörterung über die Möglichkeit "kunstwissenschaftlicher Grundbegriffe», in "Zeitschrift für Ästhetik und allgemeine Kunstwissenschaft”, 18, 1925, p. 129-161 ; trad. fr. de Pierre Rusch, Sur la relation entre l'histoire de l'art et la théorie de l'art. Contribution au débat sur la possibilité de " concepts fondamentaux de la science de l'art, "Trivium”, 6-2010 ; puis D. Cohn G. Di Liberti, Esthétique. Connaissance, Art, Expérience, Paris, Vrin, 2012, pp. 235-278.

3. E. Wind, Zur Systematik der künstlerischen Probleme, in "Zeitschrift für Ästhetik und allgemeine Kunstwissenschaft", 18, 1925, pp. 438-486.

4. Cf. A. Riegl, Historische Grammatik der bildenden Künste, aus dem Nachlaß herausgegeben von Karl M. Swoboda und Otto Pächt, Graz-Köln, Hermann Bölhaus Nachf., 1966 ; tr. fr. E. Kaufholz, Grammaire historique des arts plastiques, Paris, Klincksieck, 1978.

5. Cf. H. Wölfflin, Principes fondamentaux de l'histoire de l'art (1915), trad. par Claire et Marcel Raymond, Saint-Pierre de Salerne, Gérard Monfort éditeur, 1992

6. Je me permets ici de renvoyer à mon étude Il sistema delle arti. Storia e ipotesi, Milan, Mimesis, 2009.

7. Pour une réflexion plus large sur la performance des images cf. Alain Dierkens, Gil Bartholeyns et Thomas Golsenne (éd.), La Performance des images, Bruxelles, Éditions de l'université de Bruxelles, coll. « Problèmes d'histoire des religions » 19, 2009.

8. Cf. J. Margolis, Farewell to Danto and Goodman, "The British Journal of Aesthetics", 38, 4, 1998, pp. 353-374. Danto répondra l'année suivante (A. Danto, Indiscernability and Perception: a Reply to Joseph Margolis, "The British Journal of Aesthetics", 39, 4, 1999, pp. 321-329) et la réaction de Margolis ne tardera pas à suivre (J. Margolis, A closer Look at Danto's Account of Art and Perception, "The British Journal of Aesthetics", 40, 3, 2000, pp. 326-339). Cf. aussi J. Margolis, What is When? When is What? Two Questions to Nelson Goodman, "The Journal of Aesthetics and Art Criticism”, 39, 3, 1981, pp. 266-268.

9. Cf. Id., A closer Look at Danto's Account of Art and Perception, cit.

10. «Aesthetic perception as such has no cognitive privilege. The single large advantage of our proposal is that the problem of perception in the aesthetic domain is seen to be continuous with that of any other domain. It is merely the "use" of such perception that changes» (Id., Aesthetics Perception, "The Journal of Aesthetics and Art Criticism", 19, 2, 1960, pp. 209-213, p. 212).

11. Id., A closer Look at Danto's Account of Art and Perception, cit., p. 339.

12. Cf. Id., The Ontological Peculiarity of Works of Art, "The Journal of Aesthetics and Art Criticism", 36, 1, 1977, pp. 45-50; tr. fr., Danielle Lories ed., Philosophie analytique et esthétique, Paris, Méridiens Klincksieck, 1988, pp. 211-219, p. 218 : «Une théorie raisonnable de l'art pourrait soutenir que lorsqu'on travaille des matériaux physiques selon un certain métier artistique, il émerge culturellement un objet incarné dans les matériaux physiques qui possède un certain éventail ordonné de propriétés fonctionnelles du genre qui vient d'être mentionné. Tout objet ainsi produit peut être traité comme un artefact. C'est pourquoi les œuvres d'art existent aussi pleinement que des objets physiques, mais leur existence dépend de l'existence indépendante de 
certain objet physique. Les œuvres d'art sont donc des entités culturellement émergentes, des emblèmes-d'un-type qui existent incarnés dans des objets physiques ».

13. Cf. N. Goodman, "L'art en action", Les Cahiers du Musée National d'Art Moderne, 41, 1992, pp. 7-13.

14. J . Margolis, The Cultural Space of the Arts and the Infelicities of Reductionism, New York, Columbia University Press, 2010.

15. Id., Culture and Cultural Entities, 2nd edition, Dordrecht, Springer, 2009.

16. Id., The Arts and the Definition of the Human: Toward a Philosophical Anthropology, Stanford, Stanford University Press, 2008.

17. Pour une première orientation sur la notion d'événement dans la philosophie analytique cf. R. Casati, A. Varzi, Events, "Stanford Encyclopedia of Philosophy" (http://plato.stanford.edu/ entries/events/) ; Casati a aussi publié une très riche bibliographie raisonnée : Id., 50 Years of Events. An Annotated Bibliography 1947 to 1997 Bowling Green, Ohio, Philosophy Documentation Center, Bowling Green State University, 1997.

18. Cf. WVO Quine, Philosophy of Logic, Englewood Cliffs (NJ), Prentice-Hall, 1970.

19. Cf. R. Ingarden, "Vom formalen Aufbau des individuellen Gegenstandes", Studia Philosophica, 1, 1935, pp. 29-106.

20. La considération des faits comme des entités indépendantes est aujourd'hui plutôt répandue mais bien loin d'être unanimement partagée. À titre d'exemple, de très bons arguments contre les faits sont donnés par A. Betti, “Contre les faits”, in J. Benoist (éd.), Propositions et états de choses, Paris, Vrin, 2006, pp. 231-250.

21. J. L. Austin, P. F. Strawson, D. R. Cousin, "Symposium: Truth", in Proceedings of the Aristotelian Society, Supplementary Volumes, Vol. 24, Physical Research, Ethics and Logic, 1950, pp. 111-172. L'intervention de Strawson, La vérité, est traduite en français par J. Milner in Id., Études de logique et de linguistique, Paris, Seuil, 1977. Toujours avec le même titre Strawson avait publié en 1949 un autre article auquel Austin avait réagi en commençant ainsi la discussion (P. F. Strawon, «Truth », Analysis 6, 1949, trad. par B. Ambroise et V. Aucouturier, La vérité, in B. Ambroise et S. Laugier, éd., Philosophie du langage. Signification, vérité et réalité, Paris, Vrin, 2009, pp. 246-269). Sur ce débat, voir Ch. Alsaleh, Bruno Ambroise, "Le débat Strawson/Austin sur la vérité : théorie performative versus définition contextualiste", in J. Benoist (éd.), Propositions et états de choses, Paris, Vrin, 2006, pp. 199-230.

22. P. F. Strawson, La vérité, 1950 in Id., Études de logique et de linguistique, cit., p. 228 (la traduction d'une partie de cette citation a été modifiée pour signaler clairement la différence entre les faits et les événements).

23. Ivi, p. 224.

24. J. R. Searle, The Construction of Social Reality, London, Allen Lane-Tbc Penguin, 1995 ; trad. par C. Tiercelin, La construction de la réalité sociale, Paris, Gallimard, 1998.

25. G. E. M.Anscombe, “On Brute facts”, Analysis, 18, 3, 1958, pp. 69-72.

26. M. Ferraris Documentalità. Perché è necessario lasciar tracce, Roma-Bari, Laterza, 2009. Ferraris écrit : « Les documents au sens fort sont des inscriptions d'actes. Sous le profil d'une théorie de la société, l'ontologie des objets sociaux se présente comme documentalité, comme doctrine des documents en tant que forme la plus élevée des objets sociaux, qui sont de deux types: documents au sens fort, comme inscription d'actes, et document au sens faible, comme enregistrement de faits. Soit les documents ont des finalités pratiques, soit ils visent principalement l'évocation des sentiments. Dans ce cas, nous avons affaire avec les œuvres d'art considérées comme des choses qui font semblant d'être des personnes » (p. 361). La réflexion générale de Ferraris se présente comme une métaphysique descriptive du monde social et de l'expérience quotidienne, ontologie qui se veut clairement distincte de l'épistémologie.

27. J. Le Goff, "Documento/Monumento", in Enciclopedia, Torino, Einaudi, 1978, vol. V, pp. 38-47, p. 38 . 
28. A. Riegl, Der moderne Denkmalkultus. Sein Wesen und seine Entstehung, Wien und Leipzig, Verlage von W. Braumüller, 1903 ; trad. fr. D. Wieczorek, Le culte moderne des monuments - Son essence et sa genèse, Paris, Seuil, 1984.

29. J. Le Goff, "Documento/Monumento", cit., p. 46.

30. R. Serra, Ecrits et entretiens 1970-1989, traduit de l'américain par Gilles Courtois, Paris, Daniel Lelong éditeur, 1990. p. 159.

31. Ivi, p. 166.

32. Cf. L. Katz, “An Interview with Walker Evans”, Art in America, 59, 2, March-April 1971, après in V. Goldberg (ed.), Photography in Print: Writings from 1816 to the Present, New York, Simon and Schuster, 1981, pp. 358-69, p. 364 ; voir aussi le livre de O. Lugon, Le Style documentaire. D'August Sander à Walker Evans, 1920-1945, 2001.

33. L. Katz, “An Interview with Walker Evans”, cit., p. 363.

\section{RÉSUMÉS}

Dans notre expérience ordinaire, les faits (ce que nous savons et disons à propos des événements, nos descriptions, nos versions) sont distingués des événements, au point que leur intégration dans le catalogue du monde est controversée. Les œuvres d'art, en revanche, nous présentent à la fois une construction des faits et la possibilité d'un événement singulier. Comment une telle coexistence figure-t-elle une temporalité particulière de l'objet artistique ? De quelle manière le couple fait/événement peut-il trouver sa forme dans le couple document/monument?

In our ordinary experience, facts (that we know and say about the events, our descriptions, our versions) are clearly distinguished from events, and their inclusion in the catalogue of the world is controversial. Works of art, however, exhibit a construction of facts and the possibility of a singular event. How this coexistence sketches a particular temporality of the artistic object? How the couple fact/event can find his form by the couple document/monument?

\section{INDEX}

Mots-clés : Esthétique, ontologie de l'art, temporalité de l'œuvre d'art, fait, événement, document, monument, time capsule, Richard Serra, Walker Evans, Jochen Gerz, Esther Shalev Gerz

Keywords : Aesthetics, ontology of work of art, temporality of work of art, fact, event, document, monument, time capsule, Richard Serra, Walker Evans, Jochen Gerz, Esther Shalev Gerz

\section{AUTEUR}

\section{GIUSEPPE DI LIBERTI}

Giuseppe Di Liberti (1975) enseigne l'esthétique à l'Ecole des Beaux-Arts de Palerme. Il est membre associé du Centre d'Esthétique et Philosophie de l'Art (CEPA) de l'Université Paris 1 Panthéon-Sorbonne où il est chargé de cours en esthétique. Il a récemment publié Il sistema e le 
arti. Storia e ipotesi (Milan, Mimesis, 2009) dont la traduction française apparaîtra prochainement chez Vrin, l'édition italienne du Cabinet des beaux arts de Charles Perrault (Palerme, Centro Internazionale Studi di Estetica, 2009) et, avec Danièle Cohn, l'anthologie Textes clés d'esthétique. Connaissance, Art, Expérience (Paris, Vrin, 2012). Ses travaux de recherche portent sur le système des beaux arts, le statut de l'image, l'ontologie de l'objet artistique et l'esthétique des Lumières et notamment sur les rapports entre esthétique et physiologie chez Diderot. 